\title{
The Complex Method for Measurement Polycyclic Aromatic Hydrocarbons in Soil, Water, Plant Products
}

\author{
Natalia Tereshchenko $^{\mathrm{a}}$, Olena Khyzhan ${ }^{\mathrm{b}}$, Viktor Maksin ${ }^{\mathrm{c}}$, Kateryna Nesterova ${ }^{\mathrm{d}}$ \\ a. Candidate of Chemical Science Associate Professor of Department of Medical and General \\ Chemistry, Bogomolets National Medical University, Peremohy Ave., Kyiv 02000, Ukraine \\ b. Candidate of Chemical Science Associate Professor of Department of General, Organic and \\ Physical Chemistry, National University of Life and Environmental Sciences of Ukraine, Heroyiv \\ Oborony st., 15, Kyiv - 03041, Ukraine \\ c. Doctor of Chemical Science Professor of Department of Analytical and Bioinorganic Chemistry \\ and Water Quality, National University of Life and Environmental Sciences of Ukraine, \\ Heroyiv Oborony st., 15, Kyiv - 03041, Ukraine \\ d. Postgraduate, Department of Analytical and Bioinorganic Chemistry and Water Quality, National \\ University of Life and Environmental Sciences of Ukraine, Heroyiv Oborony st., 15, Kyiv - 03041, \\ Ukraine \\ olenakhyzhan@gmail.com
}

Keywords: polycyclic aromatic hydrocarbons, xenobiotics, chromatography, soils, water, crop products.

The measurement of xenobiotics (polycyclic aromatic hydrocarbons (PAHs) group) in agricultural objects: soil, water, plant products was developed and tested by the high performance liquid chromatography method in this work. Our investigation was carried out by using laboratory samples taken from the research farm "Snitynka". Optimal modes of high-performance liquid chromatography method with a fluorescent detector (HPLC/FLD) were established for xenobiotics measurement: benzo(a)anthracene, benzo(a)pyrene, benzo(e)pyrene, benzo(b)fluorine, chrysene and their mixtures. Metrological characteristics of PAHs measuring were established: the limit of quantitative analysis $(\mathrm{LOQ}=1.0 \mu \mathrm{g} / \mathrm{kg})$, the limit of detection $(\mathrm{LOD}=0.25 \mu \mathrm{g} / \mathrm{kg})$, measuring range $1.0-10.0 \mu \mathrm{g} / \mathrm{kg}$, the total measurement error (\%) for each xenobiotics content average did not exceed 20\%. The approbation of the methodology was performed in series of 50 model samples in the concentration range of each xenobiotics from $1.0 \pm 0.1 \mu \mathrm{g} / \mathrm{kg}$ to $10.0 \pm 0.1 \mu \mathrm{g} / \mathrm{kg}$ and in series of 50 natural samples. It is established that the developed method allows to unify laboratory control of PAHs and improves the process of monitoring analysis of xenobiotics. 


\section{Introduction}

Manufacturing of safe crop products requires control of safety indicators not only in the crop, but in a number of agricultural objects that are related to the final food product [1]. For example, crop products - oil-containing seeds, and accordingly the product of its processing - oil must be tested in a certified laboratory for a number of safety indicators, one of which is the content of polycyclic aromatic hydrocarbons (PAHs) [2,3]. It is known that according to the order of the Ministry of Health of Ukraine № 368 from 13.05.2013 "On approval of State hygienic rules and regulations" the allowable content of benzo (a) pyrene in the oil is set to not more than $2 \mu \mathrm{g} / \mathrm{kg}$ and the maximum total content of three PAHs (benzo(a)anthracene (BaA), benzo(a)pyrene (BaP), benzo(b)fluoranthene (BbF) to not more than $10 \mu \mathrm{g} / \mathrm{kg}$. It should be noted that for dietary oil and baby food oil the maximum allowable content of the xenobiotics mentioned above, including their total content, is $1 \mu \mathrm{g} / \mathrm{kg}$. The control of xenobiotics content in various objects is performed with the help of modern measuring devices. At the same time, the question of the ways of xenobiotics migration in food chains, including in agricultural production cycles, remains open. The source of PAHs xenobiotics in oilseeds is considered to be the drying process [4], but PAHs xenobiotics together with residues of plant protection products (pesticides) are contained in mature sunflower seeds, which are selected for analysis from sunflower baskets, as well as in crude oil obtained from unroasted seeds [4,5]. Contaminated seeds before the drying process contain fewer xenobiotics of the PAHs group compared to the seeds obtained after the drying process. Methods of analysis of PAHs in seeds and oil obtained from them, as a rule, are unified for convenient operation and control of pollution levels are performed by modern methods of highperformance chromatography $[4,6]$. To identify and control the paths of sunflower seeds and sunflower oil, it is necessary in the laboratory control to create a methodology that would provide quality control of at least the main objects of the food chain of sunflower oil, namely: soil and water (atmospheric and agricultural sources).

Our previous researchers have found that atmospheric water and surface water of reservoirs are contaminated with various xenobiotics, including PAHs [7]. Soils in different countries of the world accumulate a significant amount of xenobiotics, including PAHs, which are subject to biodegradation, including the cultivation of sunflower $[8,9]$. For Ukrainian-made sunflower oil and agricultural resources used to harvest oilseeds, it is desirable to conduct analysis according to a methodology that eliminates various errors and provides researchers with quality results on the levels of contamination of both soil and water. The first step in such a methodology should be the sample preparation process. According to our previous 
researches, extraction from atmospheric water of isomers of benzopyrene and other polycyclic aromatic hydrocarbons is carried out with methylene chloride [7]. This extractant can also be used to extract xenobiotics from soils and obtain oil extract from oilseeds [4-6]. To measure the PAHs content, the method of highperformance liquid chromatography with fluorescence detection (HPLC / FLD) is used, the modes of operation of which are adjusted depending on the load of analytes on the column and the presence of matrix compounds in the extract.

The purpose of this work was to study the conditions of PAHs research and develop methods for their analysis in soil, water, and crop products using high performance liquid chromatography.

\section{Experimental part}

Soil, atmospheric and reservoir water, sunflower seeds and oil samples were used, as well as model systems of all these samples, which were enriched with xenobiotics of the PAH group. The xenobiotics were extracted by three repetitions of
15 minutes macerations (total $45 \mathrm{~min}$ ), at $\mathrm{T}=18 \pm 3^{\circ} \mathrm{C}$ and constant stirring at a speed of 200 revolutions per minute. Qualification solvents "for chromatography" and "clean for analysis" were used in the work: methylene chloride, acetone, methanol, isopropanol, acetonitrile, deionized water, orthophosphoric acid, solutions of analytical PAHs standards in acetonitrile and isopropanol. Determination of the content of xenobiotics in the extract was performed by high performance liquid chromatography with fluorescence detection (HPLC / FLD). The software package Cromeleon 6.0 and the program MS Excel were used for analysis of xenobiotic analytical signals and mathematical calculations of the values characterizing the process of PAHs extraction from laboratory samples.

The technique for measuring the content of xenobiotics in the above mentioned samples consists of four main stages: homogenization of samples, obtaining an extract of xenobiotics, obtaining working solutions (replacement of the solvent), implementation of instrumental control of the content of xenobiotics (Scheme 1).

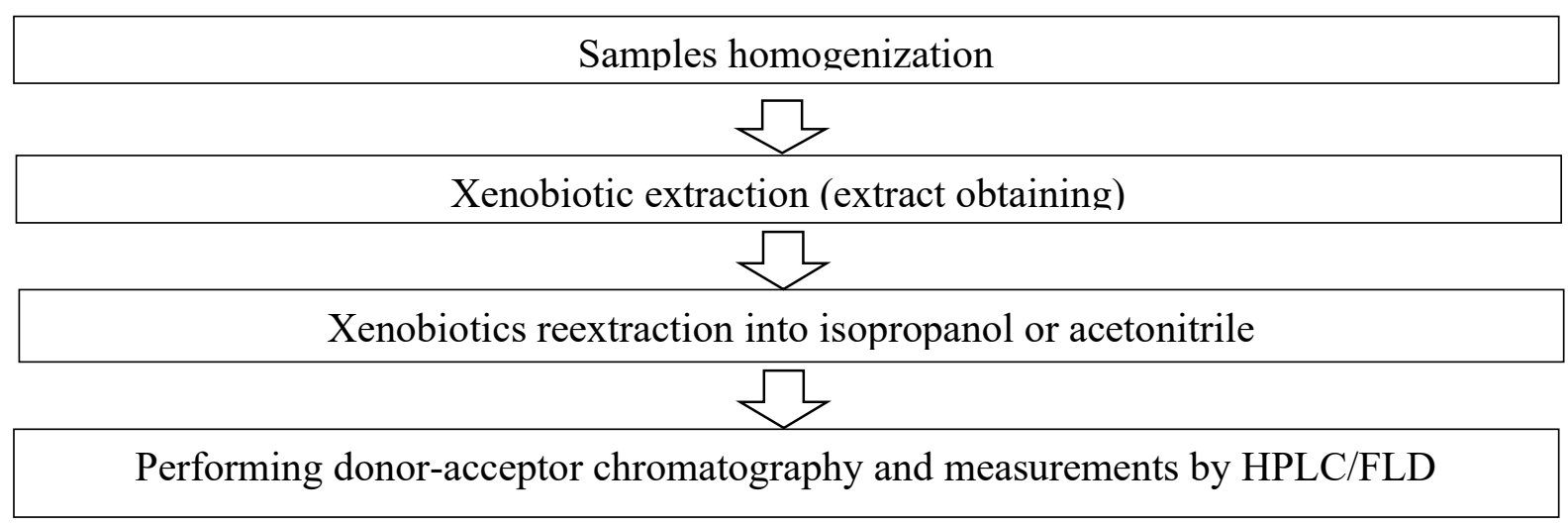

Scheme 1. Stages of samples research on the polycyclic aromatic hydrocarbons content. 
Scheme 1 illustrates the main stages of our unified methodology for measuring PAHs xenobiotics in different samples. The first stage of the methodology is the homogenization of the material. The homogenization process involves the separation of the non-target matrix from the samples. For example, silt and other mechanical impurities should be separated from the water, plant roots, fragments of stones, materials should be separated from the soil, and hard shells should be separated from seeds. The solid samples were transformed into fine and homogeneous particles by mechanical grinding. A homogeneous materials were divided on equivalent units by weight (each part was $10.0000 \pm 0.0010 \mathrm{~g}$ ). At least three equivalent units of homogeneous matrices had been used in lab experiments. Laboratory samples were placed in a flat bottom flask, mixed with an extractant in a 10:2 ratio and subjected to an extraction process by methylene chloride with constant stirring (200 revolutions per minute). Intensive stirring for 15 minutes at $\mathrm{T}=18 \pm 3^{\circ} \mathrm{C}$ contributes to the saturation of the organic extractant with xenobiotics. Methylene chloride completely dissolves the organic matrix and xenobiotics. Samples component's composition of the matrix and xenobiotics can significantly differ from one laboratory example to another.
In the structural unit of NUBiP of Ukraine, the effect of extraction conditions was investigated, which made it possible to exclude compounds (benzo(a)anthracene (BaA), chrysene (Chr), benzo(a)pyrene (BaP), benzo(e)pyrene $(\mathrm{BeP})$, benzo(b)fluoranthene $(\mathrm{BbF}))$ from the sample and carry out their measurements to establish safety sunflower seeds and oils.

Then the organic layer (methylene chloride) is separated from the matrix of the sample, and the extraction process is repeated twice more for the most complete isolation of xenobiotics.

Extraction of xenobiotics from soil, water, seeds, and oil samples under laboratory conditions also can be obtained by different standard methods. Depending on the methods of drying and subsequent heat treatment of the mixture, the qualitative and quantitative composition of xenobiotics of the $\mathrm{PAH}$ group changes; therefore, for the correct analysis of these xenobiotics in oil raw materials, thermal processes are not used [10]. The use of methylene chloride, which dissolves and removes not only the target analytes into the extract (Table 1), but also the extractive substances, plays an important role in the creation of a unified technique. 
Table 1. Results of measurement of PAHs content in samples.

\begin{tabular}{|c|c|c|c|c|c|c|}
\hline \multirow{2}{*}{ Sample code } & \multicolumn{7}{|c|}{ Xenobiotics content $\left(\mathrm{c}_{0}\right), \mu \mathrm{g} / \mathrm{kg}$} \\
\cline { 2 - 7 } & $\mathrm{BaA}$ & $\mathrm{BaP}$ & $\mathrm{BeP}$ & $\mathrm{BbF}$ & $\mathrm{Chr}$ & $\begin{array}{c}\text { The sum } \\
\text { of 5 PAHs }\end{array}$ \\
\hline Soil 1 & $12.0 \pm 0.4$ & $3.9 \pm 0.3$ & $5.2 \pm 0.2$ & $4.0 \pm 0.2$ & $7.9 \pm 0.2$ & $33.0 \pm 1.3$ \\
\hline Soil 2 & $3.9 \pm 0.1$ & $2.5 \pm 0.2$ & $3.2 \pm 0.2$ & $3.0 \pm 0.2$ & $5.1 \pm 0.2$ & $17.7 \pm 0.9$ \\
\hline Water 1 & $0.5 \pm 0.2$ & - & $1.0 \pm 0.3$ & $1.0 \pm 0.2$ & $0.5 \pm 0.2$ & $3.0 \pm 0.9$ \\
\hline Water 2 & - & - & $0.5 \pm 0.1$ & - & $0.5 \pm 0.2$ & $1.0 \pm 0.3$ \\
\hline Seeds 1 & $0.5 \pm 0.2$ & $0.9 \pm 0.3$ & $1.2 \pm 0.3$ & $1.0 \pm 0.2$ & $2.0 \pm 0.2$ & $5.6 \pm 1.2$ \\
\hline Seeds 2 & $1.3 \pm 0.2$ & $0.7 \pm 0.1$ & $1.0 \pm 0.3$ & $0.5 \pm 0.1$ & $0.9 \pm 0.2$ & $4.4 \pm 0.9$ \\
\hline Oil 1 & $1.8 \pm 0.3$ & $3.7 \pm 0.3$ & $2.9 \pm 0.4$ & $3.0 \pm 0.4$ & $5.4 \pm 0.5$ & $16.8 \pm 1.9$ \\
\hline Oil 2 & $3.4 \pm 0.4$ & $2.2 \pm 0.4$ & $3.5 \pm 0.3$ & $1.7 \pm 0.3$ & $2.9 \pm 0.3$ & $13.7 \pm 1.7$ \\
\hline
\end{tabular}

Benzo(a)anthracene (BaA), Benzo(a)pyrene (BaP), Benzo(e)pyrene (BeP), Benzo(b)fluoranthene (BbF), Chrysene

$(\mathrm{Chr})$.

The resulting solutions, after three chromatographic study shown in Table 1 were extracts, are combined and sent to the next step obtained in the study of extracts after extraction extractant removal by the evaporation method. with methylene chloride. First of all, the After evaporation of the methylene chloride, a withdrawal and replacement of methylene thin film of xenobiotics forms inside the flask, which can easily dissolve in $1.0 \mathrm{~mL}$ of chloride with isopropanol, or acetonitrile, is isopropanol or acetonitrile. We can use isopropanol, as a universal solvent for xenobiotic films of all matrices; but acetonitrile is used only for dissolving xenobiotic films extracted from water and soil.

\section{Results and discussion}

As can be seen from Table 1, the soil is the most polluted with polycyclic aromatic hydrocarbons. Their use for growing sunflower requires further research and may create prerequisites for the accumulation of these xenobiotics in culture. The results of the necessary for the measurement of xenobiotics using the method of donor-acceptor solid-phase extraction (SPE) combined with high performance liquid chromatography (HPLC/FLD). Each PAHs mixture was concentrated by drawing through the SPE column in the first minutes of the process. After this PAHs were eluted with isopropanol. The elution process was lasted for 20 minutes. The resulting isopropanol solutions were collected and were pumped through the analytical chromatography column prior to injection in detection system (HPLC/FLD method). The results are shown in Table 1. The modes of 
chromatographic separation of xenobiotics of the PAH group established by our earlier investigations allows the analysis of extracts of various objects $[4,6,7]$.
Using the program files for controlling the operation of the Dionex 3000 Dual pump HPLC / FLD device, developed for the study of PAHs mixtures, the results of chromatographic control were obtained in this work (Fig. 1, 2).

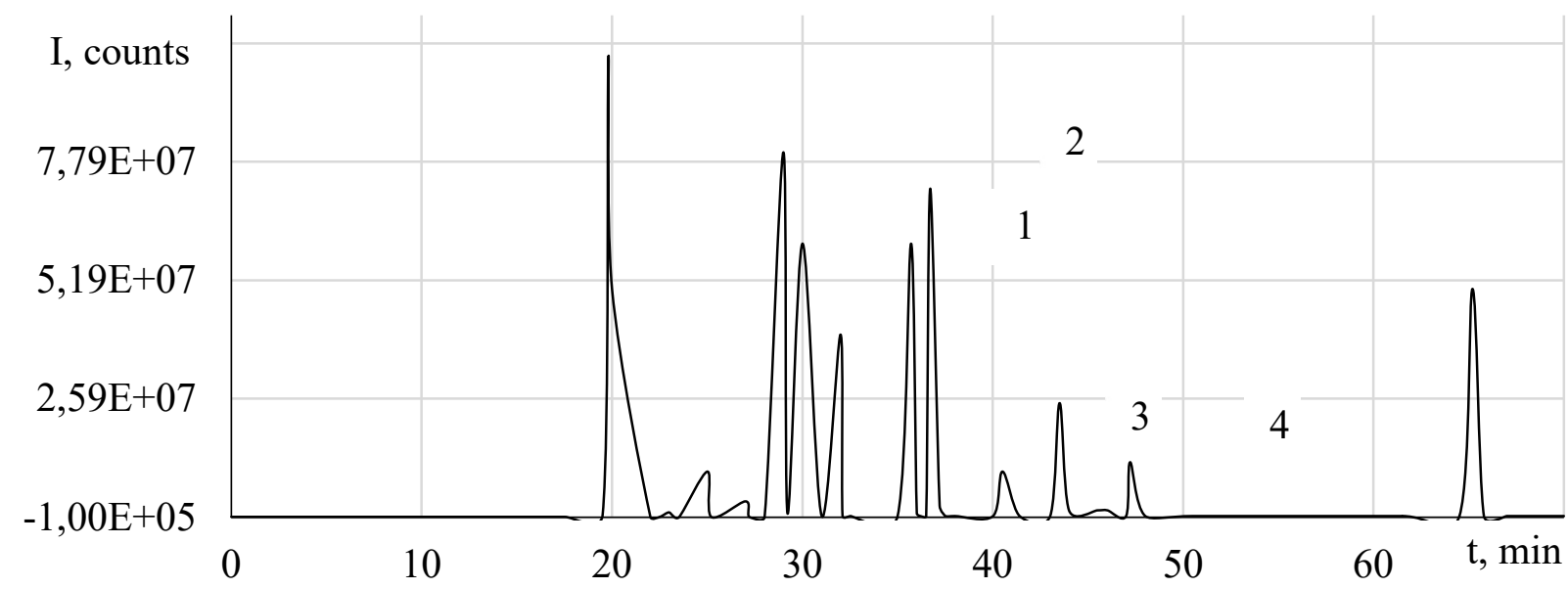

Figure 1. Chromatogram of the soil sample № 2. Peaks: 1 - Benzo(a)anthracene, 2 - Chrysene, 3 - Benzo(b)fluoranthene, 4 - Benzo(a)pyrene. HPLC / FLD chromatograph, Dionex 3000. Mobile phase A: isopropanol; Mobile phase B: acetonitrile-water. Gradient elution. Injection $40 \mu 1$

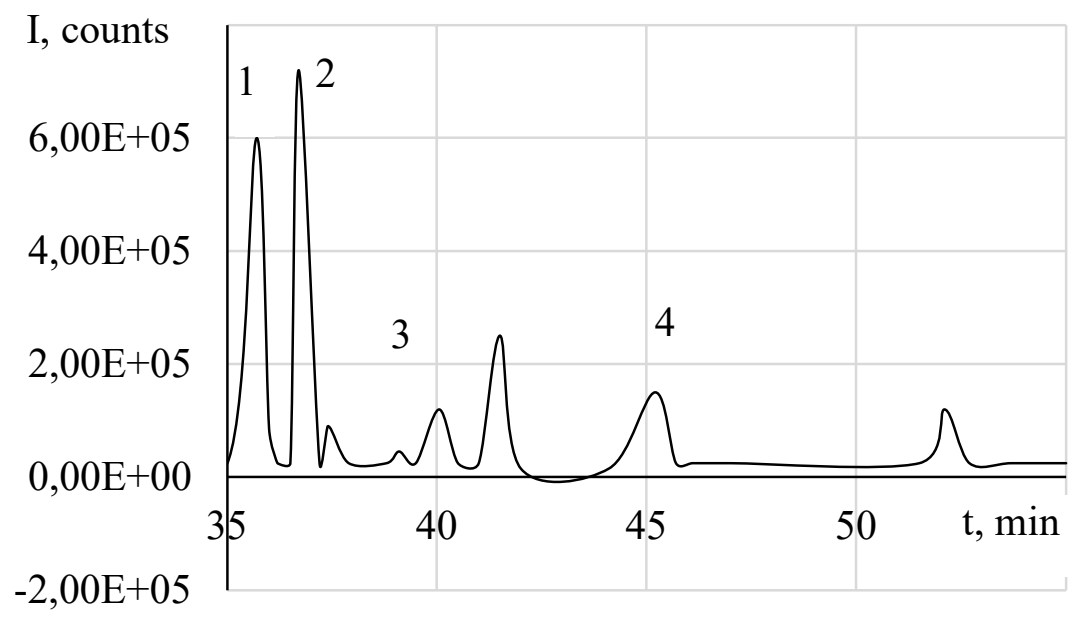

Figure 2. Chromatogram of the oil sample № 2. Peaks: 1 - Benzo(a)anthracene, 2 - Chrysene, 3 - Benzo(b)fluoranthene, 4 - Benzo(a)pyrene. HPLC / FLD chromatograph, Dionex 3000. Mobile phase A: isopropanol; Mobile phase B: acetonitrile-water. Gradient elution. Injection $40 \mu 1$ 
As can be seen from Figure 1, the sample contains not only significant amounts of target PAHs, but also other compounds of the PAH class, the identification of which and quantitative analysis should be performed in subsequent studies.

To create a methodology for the analysis of samples with a total content of xenobiotics, model systems were created, and the studied samples were subjected to the process of artificial enrichment with xenobiotics.

Metrological characteristics of PAHs measuring were established: the limit of quantitative analysis (LOQ), the limit of detection (LOD) (Fig.3). Figure 3 contains information on linear regression, set in the range from 1.0 to $10.0 \mu \mathrm{g} / \mathrm{kg}$. The first point of quantitative analysis is the concentration of 1.0 $\mu \mathrm{g} / \mathrm{kg}$, the presence of xenobiotic's trace amounts was observed in the chromatogram as a peak with using the model solution enriched with a concentration of $0.25 \mu \mathrm{g} / \mathrm{kg}$. Samples in which the xenobiotic content was greater than $10 \mu \mathrm{g} / \mathrm{kg}$ were diluted 1.5 to 2 times by the pure solvent. The dilution factor was taken into account in the calculating (calculation formula 1):

$C_{P A H}=C_{\text {measured }} \times 100 \times k$, where is

$C_{P A H^{-}}$- content in sample,

$C_{\text {measured }}$ - content in HPLC/FLD analysis, calculated using a calibration curve $\mathrm{k}$ - dilution factor.

The stability of the results (examined in series of more than 200 experiments with a confidence level of $\mathrm{P}=0.95$, the total measurement error (\%) for each xenobiotics content average did not exceed 20\% (Table 2). It was found that all compounds of the PAH group introduced into the samples are removed during maceration with methylene chloride. For laboratory soil samples, sunflower seeds and sunflower oil, artificially enriched with PAHs, it is characteristically that the concentration of PAHs is set to be greater than the applied concentration of xenobiotics, the percentage of xenobiotics withdrawal exceeding 100\% (Table 2). Such results of quantitative analysis may be due to the fact that xenobiotics were present in the samples before the enrichment process, their amount determined by chromatographic control has a certain error in the range from 10 to $20 \%$, thus, when calculating the percentage of recovery, there was an increase in the error value characterizing the number of xenobiotics and their sums in the test sample. Attention is drawn to the results of PAHs content in artificially enriched water samples. The percentage of PAHs return from artificially enriched samples is less than $100 \%$, which indicates the loss of xenobiotics during sample preparation and chromatographic control. The results shown in Table 2 indicate that for lipophilic samples with a certain background level of contamination with compounds of the PAH group, quantitative analysis provides overestimated results of measuring the PAH content, and for lipophobic samples, quantitative analysis is accompanied by 
a loss of analytes at the stages of sample method for measuring the residual amounts of preparation, re-extraction and chromatographic xenobiotics by the methods of modern analysis. At the same time, the total error of the chromatography [11]. proposed methodology does not exceed $20 \%$ the maximum value of the total error of the

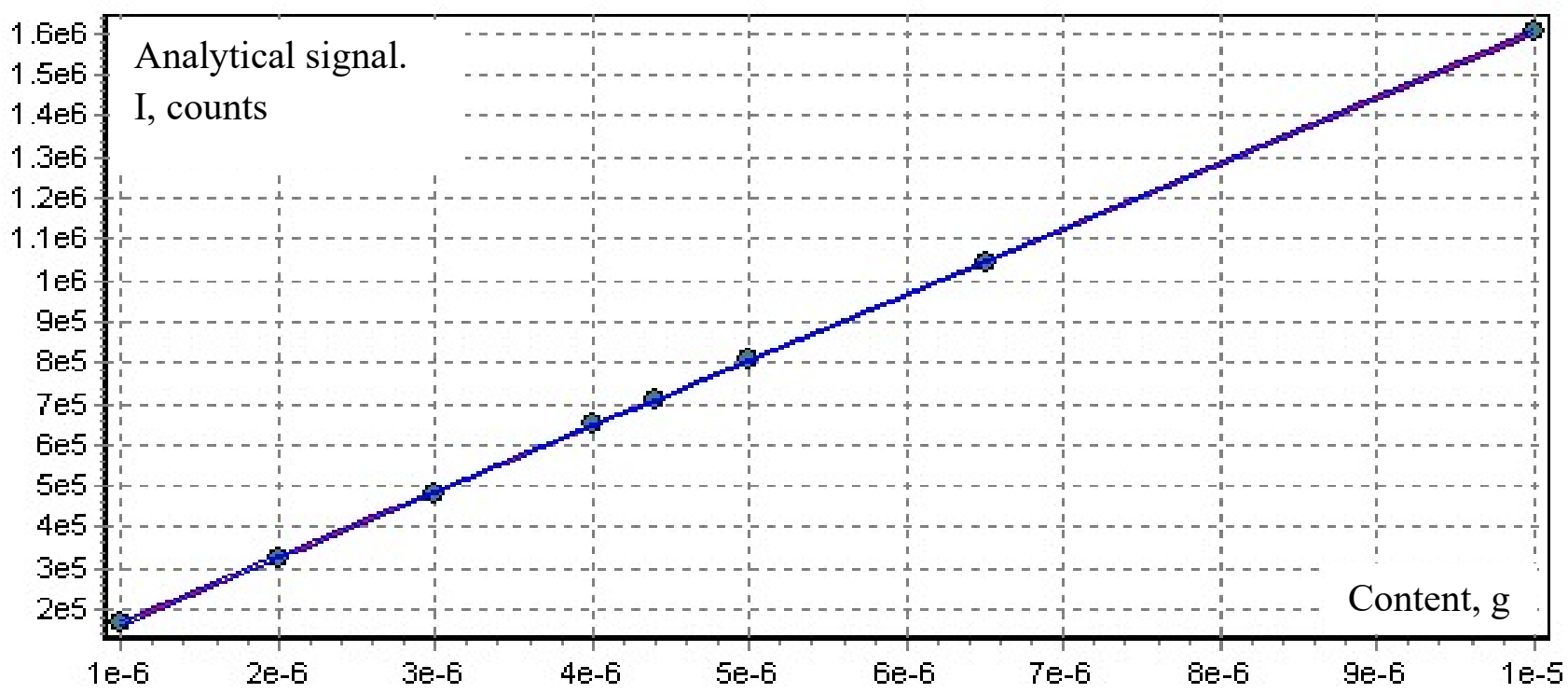

Figure 3. Example of statistical analysis result (Spline program) of benzo(e)pyrene content measurement in working solution, 10 sunflower seeds extracts from 10 parallel model seeds system)

Table 2. The PAHs measurement results in samples artificially enriched with PAHs.

\begin{tabular}{|c|c|c|c|}
\hline \multicolumn{4}{|c|}{ Soil sample 3 with artificial enrichment (injected $C_{5 P A H}=17.82 \pm 1.09 \mu \mathrm{g} / \mathrm{kg}$ ) } \\
\hline Compound name & Content, $\mu \mathrm{g} / \mathrm{kg}$ & Extraction, \% & $\begin{array}{c}\text { Total error of the } \\
\text { measuring method, } \\
\%\end{array}$ \\
\hline Benz (a) anthracene & 4.67 & 107.9 & 15 \\
\hline Benz (a) pyrene & 5.06 & 107.3 & 18 \\
\hline Benz (e) pyrene & 2.01 & 102.0 & 18 \\
\hline Benz (b) fluoranthene & 4.89 & 106.9 & 20 \\
\hline Chrysen & 4.95 & 102.4 & 15 \\
\hline Sum of 5 PAHs & 21.58 & 121.2 & 17 \\
\hline \multicolumn{4}{|c|}{ Water sample 3 with artificial enrichment (injected $C_{5 P A H}=5.99 \pm 0.73 \mu \mathrm{g} / \mathrm{kg}$ ) } \\
\hline Compound name & Content, $\mu \mathrm{g} / \mathrm{kg}$ & Extraction, \% & $\begin{array}{c}\text { Total error of the } \\
\text { measuring method, } \\
\%\end{array}$ \\
\hline Benz (a) anthracene & 1.02 & 97.9 & 19 \\
\hline Benz (a) pyrene & 1.36 & 99.3 & 22 \\
\hline Benz (e) pyrene & 0.99 & 98.9 & 14 \\
\hline Benz (b) fluoranthene & 1.52 & 98.5 & 17 \\
\hline Chrysen & 1.02 & 99.3 & 15 \\
\hline Sum of 5 PAHs & 5.91 & 98.65 & 17.5 \\
\hline \multicolumn{4}{|c|}{ Seeds sample 3 with artificial enrichment (injected $C_{5 P A H}=5.99 \pm 0.73 \mathrm{mcg} / \mathrm{kg}$ ) } \\
\hline
\end{tabular}


FRENCH-UKRAINIAN JOURNAL OF CHEMISTRY (2021, VOLUME 09, ISSUE 02)

\begin{tabular}{|c|c|c|c|}
\hline Compound name & Content, $\mathrm{mcg} / \mathrm{kg}$ & Extraction, \% & $\begin{array}{c}\text { Total error of the } \\
\text { measuring method, } \\
\%\end{array}$ \\
\hline Benz (a) anthracene & 1.22 & 117.8 & 20 \\
\hline Benz (a) pyrene & 1.64 & 120.1 & 19 \\
\hline Benz (e) pyrene & 1.75 & 113.5 & 18 \\
\hline Benz (b) fluoranthene & 1.08 & 108.7 & 16 \\
\hline Chrysen & 1.17 & 114.3 & 16 \\
\hline Sum of 5 PAHs & 6.86 & 114.5 & 17.8 \\
\hline \multicolumn{4}{|c|}{ Oil sample 3 with artificial enrichment (injected $C_{5 P A H}=5.99 \pm 0.73 \mathrm{mcg} / \mathrm{kg}$ ) } \\
\hline Compound name & Content, $m c g / k g$ & Extraction, \% & $\begin{array}{c}\text { Total error of the } \\
\text { measuring method, } \\
\%\end{array}$ \\
\hline Benz (a) anthracene & 1.18 & 113.9 & 20 \\
\hline Benz (a) pyrene & 1.72 & 125.9 & 19 \\
\hline Benz (e) pyrene & 1.70 & 110.3 & 18 \\
\hline Benz (b) fluoranthene & 1.11 & 111.7 & 16 \\
\hline Chrysen & 1.14 & 111.4 & 16 \\
\hline Sum of 5 PAHs & 6.85 & 114.4 & 17.8 \\
\hline
\end{tabular}

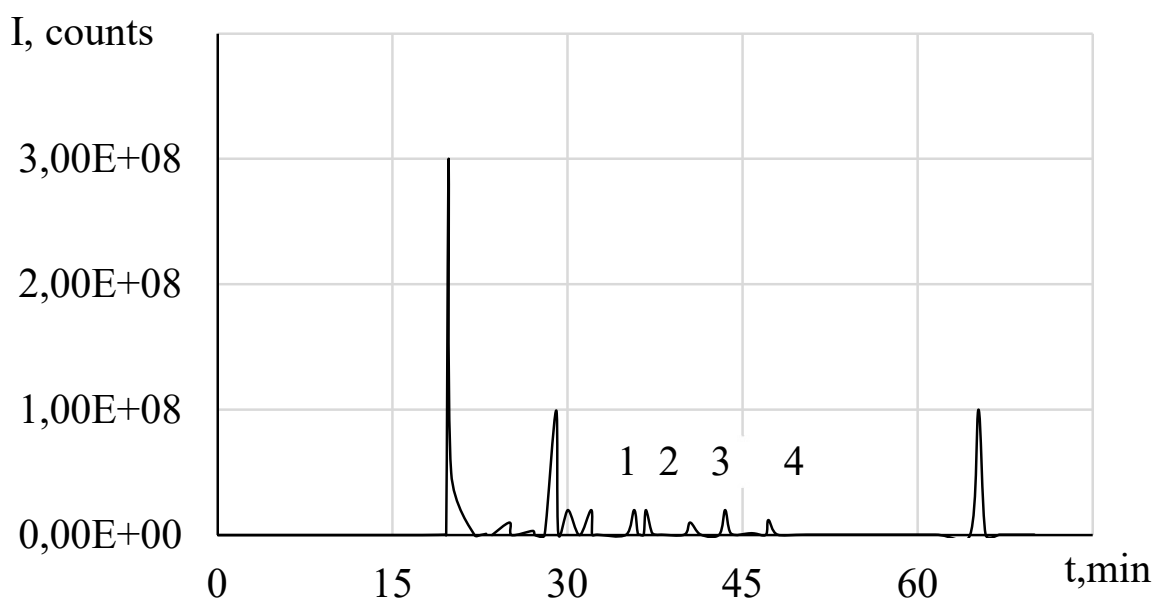

Figure 4. Chromatogram of xenobiotic extract from surface water enriched with a mixture of analytical standards of xenobiotics. Peaks: 1 - Benzo(a)anthracene, 2 - Chrysene, 3 - Benzo(b)fluoranthene, 4 - Benzo(a)pyrene. HPLC/FLD chromatograph, Dionex 3000. Mobile phase A: isopropanol; Mobile phase B: acetonitrile-water. Gradient elution. Injection $40 \mu \mathrm{l}$

The optimal parameters of the shows the example of chromatogram and methodology were used in the analysis of a series of 50 samples represented by different matrices: xenobiotics measurement.

It should be noted, that natural water soil, water for irrigation, oilseeds. Figure 4 samples are less contaminated with PAH xenobiotics than natural soil samples, and before 
their chromatographic analysis it is necessary to perform repeated concentrations of PAH extracts by the methylene chloride from series of parallel samples of one water object. The analysis of natural water samples is more difficult than the analysis of other samples. The number of parallel extractions can be up to 30-35 repetitions. However, there is an easier way to prepare extracts from an aliquot volume of $1 \mathrm{~L}(1 \mathrm{~kg})$ the extraction in a separatory funnel. This method of extraction lasts 90 minutes and allows constant uniform mixing of the sample-extractant system. Liquid matrices with $1 \mathrm{~L}$ volume, contains PAHs, were analyzed using this method. In this case we had not used the dilution factor in calculations by formula 1 . The calculation of PAHs in liquids were not complicated by the need to determine a dilution factor. Xenobiotics contents were calculated for parent matrices in the units $\mathrm{mkg} / \mathrm{L}(\mathrm{mg} / \mathrm{L})$.

\section{Conclusions}

Thus, the methodology for measuring the PAH content in soils, water, seeds and sunflower oil, developed and tested in this work, provides an analysis of the list of five PAH compounds. The application of this methodology is a convenient tool for monitoring PAHs contents; the unification of the sample preparation procedure reduces the duration of the analysis in general. Due to the application of the method of solid-phase extraction of xenobiotics (SPE), an increase in the selectivity of the method was achieved; measurements were carried out by chromatographic analysis with a fluorescent detector with an error not exceeding $20 \%$.

\section{References}

[1] Ukraine. Verkhovna Rada of Ukraine. On basic principles and requirements for food safety and quality. Law of Ukraine № 771/97-VR. Kyiv: Verkhovna Rada of Ukraine; 1997.

http://search.ligazakon.ua/1_doc2.nsf/link1/ed_2011_02_0 3/Z970771.html

[2] U.S. Environmental Protection Agency. Toxicological Review of Benzo[a]pyrene. Review of EPA's Draft Assessment. Washington: U.S. Environmental Protection Agency; 2017.

[3] ISO 9001:2008, Quality management systems Requirements [Internet]; 2008 [last modified 2009 July]. Available from: https://www.iso.org/standard/46486.html [4] Hrybova N. Xenobiotics of PAHS group is extracted from sunflower seeds. Scientific Herald of National University of Life and Environmental Sciences of Ukraine. Series: Agronomy. 2018; 294:209218. https://doi.org/10.31548/agr2018.294.209

[5] Melnychuk S, Lohanska V, Baranov Y, Zemtsova O, Maksymchuk I, Hribova N. Monitoring in oils pesticides residues and polycyclic aromatic hydrocarbons for safety of vegetable oils. Potravinarstvo: Scientific Journal for Food Industry. 2013; 7: 45-52.

[6] Nesterova L, Hrybova N, Khyzhan O, Ushkalov V. Development of controls method for the isomers of polycyclic aromatic hydrocarbons in vegetable oils. Scientific Journal of National University of Life and Environmental Sciences of Ukraine. Series: Agronomy. 2018; 286:312-320.

[7] Grybova N, Nesterova L, Khyzhan O, Ushkalov V, Maksin V. Determination of Polycyclic Carbohydrates in Atmospheric Water by the Method of Chromatography. Journal of Water Chemistry and Technology 2018;40(5): 297-301. https://doi.org/10.3103/s1063455x18050089 
[8] Zafra G, Taylor TD, Absalón AE, CortésEspinosa DV. Comparative metagenomic analysis of $\mathrm{PAH}$ degradation in soil by a mixed microbial consortium. Journal of hazardous materials. 2016; 318: 702-710.

[9] Tejeda-Agredano MC, Gallego S, Vila J, Grifoll M, Ortega-Calvo J J, Cantos M. Influence of the sunflower rhizosphere on the biodegradation of PAHs in soil. Soil Biology and Biochemistry. 2013; 57: 830-840.

[10] Kobervein YuM. About the problem of the accumulation in sunflower seeds 3,4-benzpyrene during drying. Fat-and-oil industry. 1976; 3: 17-19.

[11] European Commissionhealth \& Consumer Protection Directorate-General. Guidance document on analytical quality control and validation procedures for pesticide residues analysis in food and feed. Document No SANCO/12495/2011. EU: European Commissionhealth \& Consumer Protection Directorate-General; 2013. 